\title{
The significance of Cancer stem cell markers' gene expression and Relevance for Survival Outcomes
}

\author{
Kevin Dzobo ${ }^{1,2^{*}}$, Dimakatso Alice Senthebane ${ }^{1,2^{*}}$, Chelene Ganz ${ }^{1,2 \#}$ and Nicholas Ekow \\ Thomford $^{3,4}$
}

${ }^{1}$ International Centre for Genetic Engineering and Biotechnology (ICGEB), Cape Town Component, Wernher and Beit Building (South), UCT Medical Campus, Anzio Road, Observatory 7925, Cape Town, South Africa. kdzobosnr@yahoo.com (K.D); dimakatsosenthebane@gmail.com (D.A.S); cheleneganz@gmail.com (C.G)

${ }^{2}$ Division of Medical Biochemistry and Institute of Infectious Disease and Molecular Medicine, Department of Integrative Biomedical Sciences, Faculty of Health Sciences, University of Cape Town, Cape Town, South Africa

${ }^{3}$ Division of Human Genetics, Department of Pathology \& Institute for Infectious Disease and Molecular Medicine, Faculty of Health Sciences, University of Cape Town, Anzio Road, Observatory 7925, Cape Town, South Africa. n.e.thomford@ uccsms.edu.gh (N.E.T).

${ }^{4}$ Department of Medical Biochemistry, School of Medical Sciences, College of Health Sciences, University of Cape Coast, PMB, Cape Coast, Ghana

*Contributed equally

*Correspondence: kdzobosnr@yahoo.com ; Tel: +27 842953708

\begin{abstract}
Solid tumors display complex biology and most therapies including chemotherapy cannot prevent therapy resistance and relapse. Most therapeutics target cancer cells, but recent data suggest the presence of cancer stem cells as cells with self-renewal and tumorigenic abilities. Cancer stem cell markers have been suggested to have prognostic value and can be targeted during cancer treatment and in resistant disease. CSCs have been postulated to play significant contextual roles in tumor initiation, progression, therapy resistance and metastasis. CSCs have thus been targeted by new generation cancer drugs. The transcriptional expression of several CSC markers in different cancers was evaluated by searching publicly available The Cancer Genome Atlas (TCGA) and Gene Expression Profiling Interactive Analysis (GEPIA) databases. We report here new findings on expression and prognostic significance of CSC markers in several cancers by examining the expression of CSCs markers in tumor tissues versus the adjacent normal tissues. We found that CSC markers were mostly highly expressed various tumors such as colon, lung, pancreatic and esophageal cancers. No CSC marker is expressed in the same pattern in all cancers and individual CSC marker expression was not linked to patient survival. This analysis calls for continued research on CSCs and clinical evaluation of the CSC markers in relation to prognosis of cancers in large population samples. Novel cancer drugs ought to target CSCs, cancer cells and tumor microenvironment variations.
\end{abstract}

Keywords: tumor microenvironment, biomarkers, solid cancers, computational biology, cancer stem cells, anti-cancer stem cell therapy, chemotherapy. 


\subsection{Introduction}

Huge progress has been made in the treatment of cancer but its health burden continues to increase globally, raising the need for novel therapeutic strategies to contain cancer $[1,2]$. Whilst current anti-cancer strategies target genetic and epigenetic changes in cancer cells, most of these have proved to be unsuccessful [3-7]. Detailed analyses of tumors have revealed the important role the tumor microenvironment (TME) play in tumor maintenance and therapy resistance [8-14]. Within the tumor microenvironment are several stromal cells and the extracellular matrix (ECM) that contributes to tumor response to therapies and metastasis $[5,6,15]$.

For many cancers, clinical factors, such as tumor stage and differentiation, have been reported to be associated with prognosis of the disease $[5,6,16-22]$. Many times the utilisation of these clinical factors is not enough for risk stratification and prediction of disease outcome. This can result in wrong clinical prognosis predictions. For many cancers, including those that are not well studied such as esophageal cancer, there is an urgent need for the identification of reliable prognostic factors that can accurately predict clinical prognosis. Several studies have shown that the prognostic value of cancer stem cells depends on the type of cancer and the histological subtype [23-28]. In addition, several recent studies have revealed that a major mechanism for post-therapeutic recurrence and metastasis of cancers is the presence of therapy-resistant cancer stem cells [5, 6, 29-31]. It has been demonstrated that chemoresistance to 5-fluorouracil and cisplatin in several cancer patients may occur through the increased expression of microRNAs such as miR-200 and cancer stem cell-related proteins $[32,33]$. Furthermore, increased expression of the multidrug resistance 
protein 2 (MRP2) has been shown in the tissue samples of patients resistant to neo-adjuvant chemotherapy including 5-fluorouracil, doxorubicin and cisplatin [34-36].

Thus the focus of many studies has been on a subpopulation of cells with stem cell-like characteristics called cancer stem cells (CSCs) [5, 6, 37]. CSCs have been identified in many cancers such as breast, glioma, melanoma, ovarian, head and neck cancer [5, 38-45]. Besides being important in the initiation, maintenance and relapse of tumours, CSCs have been shown to modify neoplastic cell behaviour and aggressiveness as well as therapeutic response. CSCs are rare tumour cells with the ability to self-renew and can proliferate extensively. They also have the ability to resist chemotherapy and radiotherapy treatments [5, 38, 40-42, 46-49]. These cells can form tumour-spheres in vitro and have been shown to be enriched for tumorigenic cells by their ability to form xenograft tumours in severe combined immunodefficient (SCID) mice [50-54]. CSCs can be isolated through various methods including the use of antibodies against various surface markers such as cluster of differentiation 44 (CD44), CD24, CD133, and CD166 [46, 53-57]. Many studies have shown that CD44+/CD24- phenotype is associated with a worse prognosis in many cancers including breast cancer [58-61]. Other markers such as CD90, aldehyde dehydrogenase 1 (ALDH1), EpCAM and p63 have also shown to be useful in this regard [62, 63]. Besides the use of antibodies, CSCs can be isolated and identified via the use of side population cells. Side population cells in tumours are a small subpopulation of cancer cells with stem cell-like properties and can be isolated and identified by dual wavelength FACS analysis [56, 57, 6466]. In several cancers, it has been shown that the side population tends to enrich CSCs and can be isolated using Hoechst 33342 dye [64-66]. 
Overall, CSCs are an emerging target for cancer therapy and any therapy targeting CSCs hold great potential for improving cancer treatment and outcome. In this study we performed a bioinformatic analysis to determine the prognostic value of CSC markers in several cancers. We utilized the publicly available databases The Cancer Genomic Atlas (TCGA) (http://cancergenome.nih.gov) and Gene Expression Profiling Interactive Analysis (GEPIA) (http://gepia.cancer-pku.cn) to determine CSC markers' (ABCB1, ABCG2, ALDH1, CD24, CD44, CD90, CD133, CXCR4, EPCAM, ICAM1 and NESTIN) expression in tumor tissues versus the adjacent normal samples and in relation to patients' overall survival. Our analysis reveal that whilst CSC markers' expression is upregulated in tumor tissues versus normal samples, individual CSC markers expression may not be associated with overall patients' survival.

\subsection{Materials and Methods}

\subsection{Bioinformatic Analysis of CSC markers' RNA-seq analysis}

This study utilised the publicly available TCGA and GEPIA databases and did not involve human subject recruitment nor animal studies. Whole genome messenger RNA expression levels of $A B C B 1, A B C G 2, A L D H 1, C D 24, C D 44, C D 90, C D 133, C X C R 4, E p C A M, I C A M 1$ and NESTIN were examined in tumor and normal adjacent tissues (Match TCGA normal and GTEx data) in relation to patients' survival outcomes. TCGA and GEPIA data was accessed for different cancers in March 2020 via the respective portals. The GEPIA website contains web-based tools that allow in-depth analysis of the CSC markers expression within the TCGA and GEPIA databases. 


\subsection{Statistical Analyses}

Statistical analyses were performed using GraphPad Prism software (version 6; San Diego, USA). In addition, significance of differences was tested by Student's t-test and one way analysis of variance (ANOVA) test. Statistical significance between groups regarding overall survival was performed by the Kaplan-Meier analysis with the log-rank test (95\% confidence interval). GEPIA use statistical analysis that divided patients into high and low expression of CSC markers. The same number of patients for each group was used to analyze survival chance. Statistical significant was set as $P<0.05$.

\subsection{Results}

\subsection{Significance of Cancer stem cells in tumorigenesis}

\subsubsection{Colon Cancer}

One of the most deadly cancers, colorectal cancer, has been reported to contribute above $10 \%$ of diagnosed cancers worldwide and causes approximately $9 \%$ of all cancer deaths each year $[67,68]$. The incidence of colorectal cancer is increasing globally and patients' survival varying greatly among countries. Intense research continues to unravel the biology and mechanism of progression of colorectal cancer [69].

Bioinformatic analysis showed that CSC markers, CD24, CD44, CD90 and CD133 expression was significantly enhanced in colon adenocarcinoma (COAD) compared to adjacent normal tissues (Figure 1). Surprisingly, another CSC marker, ALDH1 expression was significantly reduced in tumor samples versus normal samples (Figure 1). Consistent with the above results, 10 out of 12 colorectal cancer specimens showed medium to high 
CD44 protein expression based on immunohistochemistry-based data available at Human Protein Atlas database (www.proteinatlas.org). In the same vein, 12 out of 12 colorectal cancer specimens showed medium to high CD133 protein expression based on immunohistochemistry-based data available at Human Protein Atlas database. However, association analyses of CSC markers' expression with prognosis of COAD revealed that there were no significant differences in survival between patients expressing high CSC markers compared to patients expressing low levels of the same CSC markers (Figure 1).

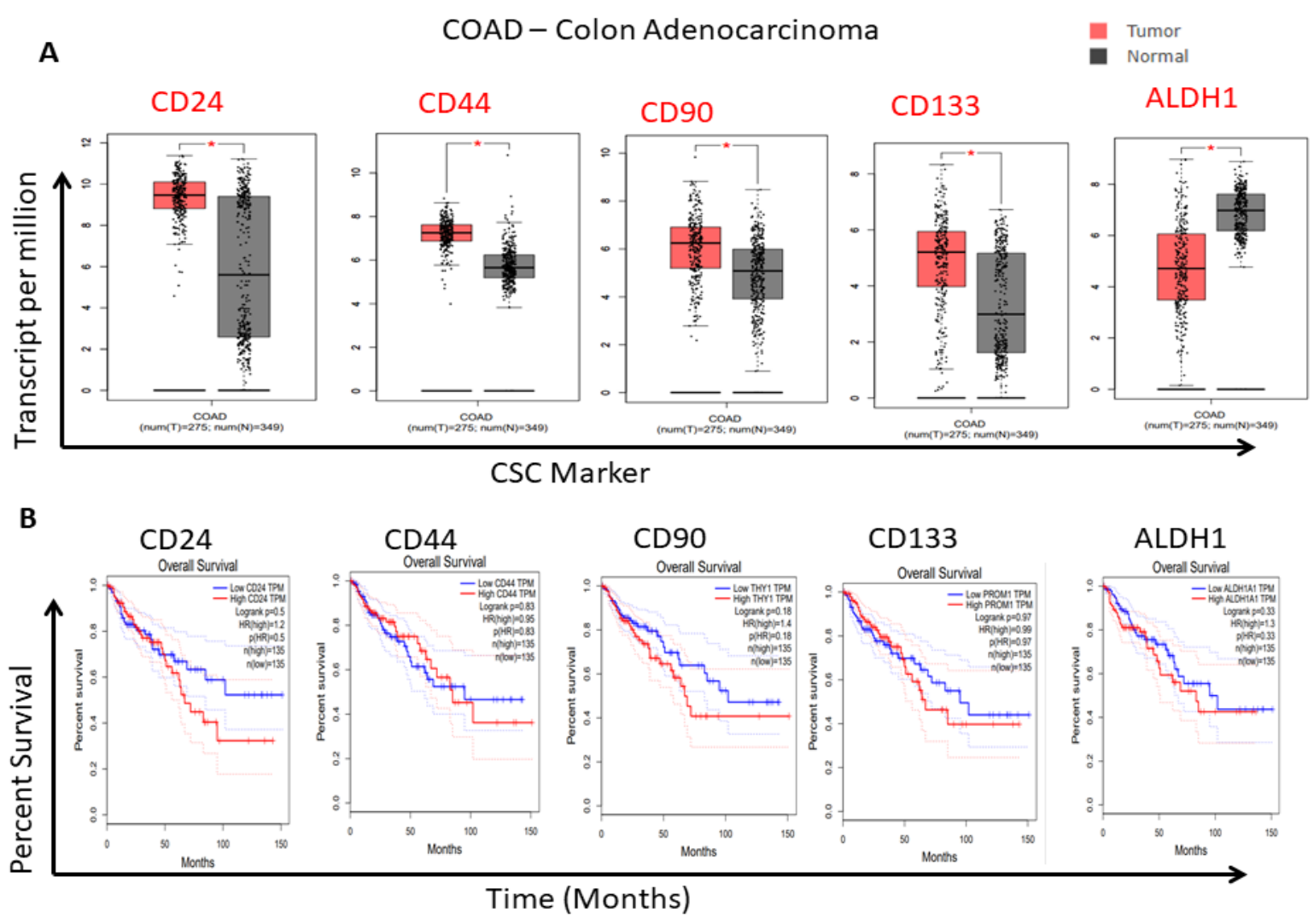

Figure 1. (A) Cancer stem cell markers' gene expression profiles in colon adenocarcinoma (COAD). The expression of CSC markers CD24, CD44, CD90, CD133 and ALDH1 in colon adenocarcinoma tissues and adjacent normal tissues (Box plot) based on TCGA/GEPIA database. Data is based on TCGA COAD samples $n=275$; normal $=349$. CSC markers showing significant differences between tumor and normal samples are shown in red with * indicated for $\mathrm{p}<0.05$ (B) Kaplan-Meier overall survival analysis of COAD patients by CSC markers expression in TCGA data set (high expression $(n=135)$, low expression $(n=135)$ ). 


\subsubsection{Lung cancer}

The most commonly diagnosed cancer globally is lung cancer and its incidence reported to be around $11.6 \%$ of the total cases of cancer [1]. Lung cancer results in more cancer deaths than any other cancer, accounting for around $18.4 \%$ of the total cancer deaths [1]. Several risk factors have been identified and include exposure to chemicals, tobacco, smoke and asbestos $[4,67,70,71]$.

With the exception of $C D 133$ and $A B C G 2$ expression, which showed similar expression in lung adenocarcinoma (LUAD) tissues versus controls, the expression of CSC markers, CD24, $C D 90$ and EpCAM was significantly upregulated in TCGA LUAD samples compared to adjacent normal samples (Figure 2). Consistent with the above results, 10 out of 12 lung cancer specimens showed medium to high EpCAM protein expression based on immunohistochemistry-based data available at Human Protein Atlas database (www.proteinatlas.org). Analysis of association of CCS markers expression with prognosis of LUAD cancer patients revealed no significant difference in overall survival between LUAD cancer patients expressing low and high CSC markers (Figure 2). 
A

LUAD- Lung Adenocarcinoma

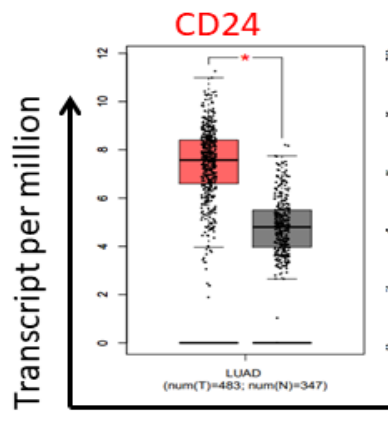

CD90
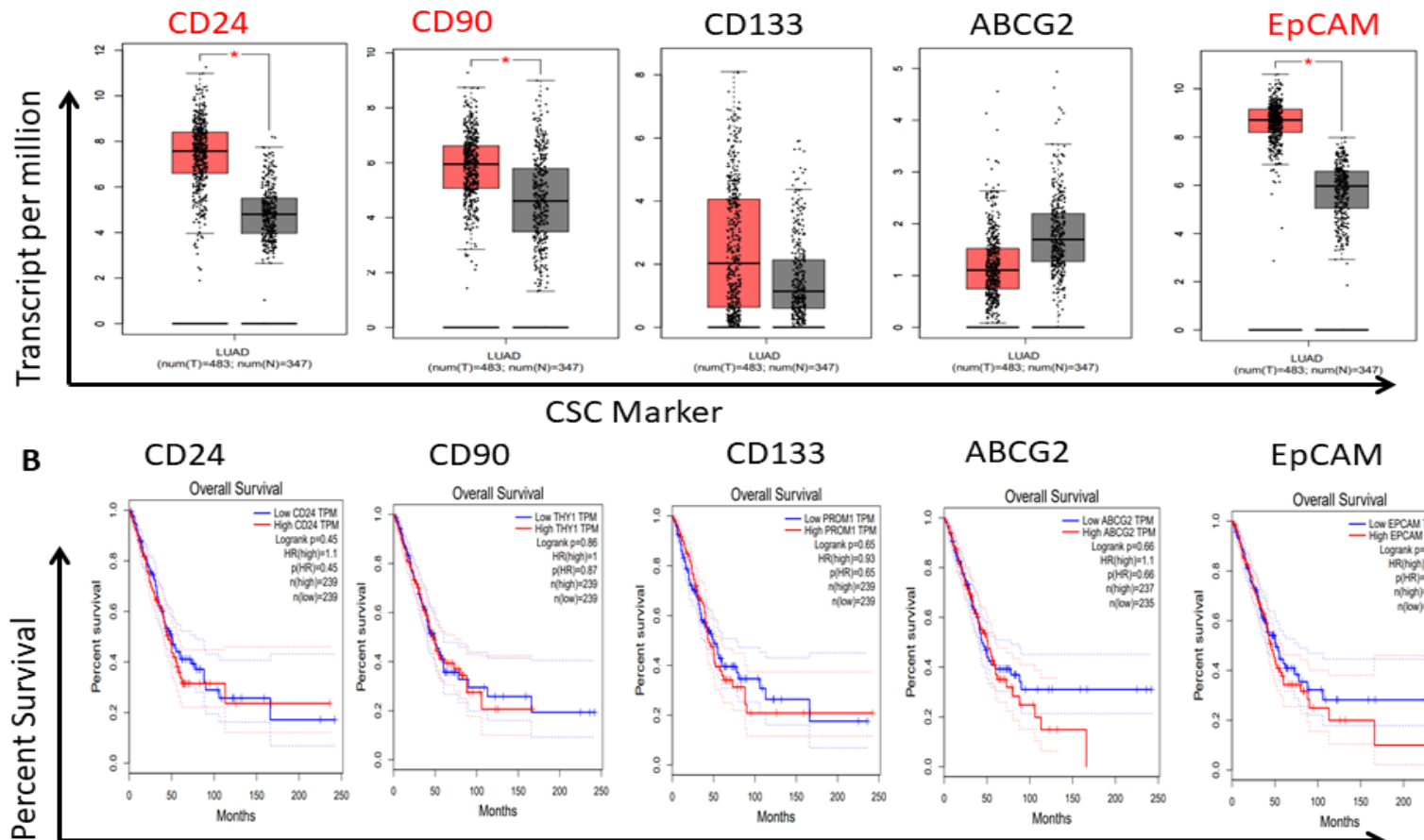

ABCG2
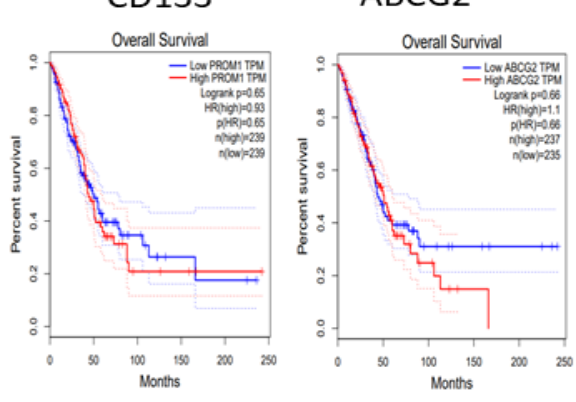

EpCAM

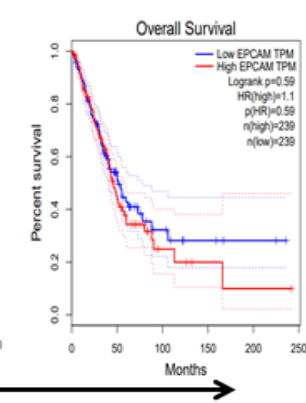

Time (Months)

Figure 2. Cancer stem cell markers' gene expression profiles in lung adenocarcinoma (LUAD). The expression of CSC markers CD24, CD90, CD133, ABCG2 and EpCAM in lung adenocarcinoma tissues and adjacent normal tissues (Box plot) based on TCGA/GEPIA database. Data is based on TCGA LUAD samples $n=483$; normal $=347$. CSC markers showing significant differences between tumor and normal samples are shown in red with * indicated for $\mathrm{p}<0.05$ (B) Kaplan-Meier overall survival analysis of LUAD patients by CSC markers expression in TCGA data set, high expression $(n=239)$, low expression $(n=239))$.

\subsubsection{Pancreatic Cancer}

One of the most lethal cancers is pancreatic cancer with estimates showing that above 338,000 people were diagnosed with the disease worldwide in 2012 [72, 73]. One of the major histological subtypes is pancreatic ductal adenocarcinoma and show aggressive growth resulting in high mortality rate $[74,75]$. Pancreatic adenocarcinoma (annotated as PAAD within TCGA and GEPIA databases) is the most common pancreatic cancer, with estimates showing that 9 out of 10 people with pancreatic cancer have this type of cancer [76-78]. 
Using the TCGA database samples, our bioinformatic analysis showed that, $A B C B 1, C D 133$, CXCR4, EpCAM and NESTIN expression was significantly upregulated in pancreatic adenocarcinoma (PAAD) tumor samples versus adjacent normal samples (Figure 3). In agreement with the above results, 9 out of 12 pancreatic cancer specimens showed medium to high CD133 protein expression based on immunohistochemistry-based data available at Human Protein Atlas database (www.proteinatlas.org). Assessment of the association of CSC markers expression with prognosis of PAAD cancer patients revealed no significant difference in overall survival between PAAD cancer patients expressing low and high CD133, CXCR4, EpCAM and NESTIN CSC markers (Figure 3). Low expression of ABCB1 was associated with low survival in PAAD patients (log rank $\mathrm{p}=0.0059$ ) (Figure 3).

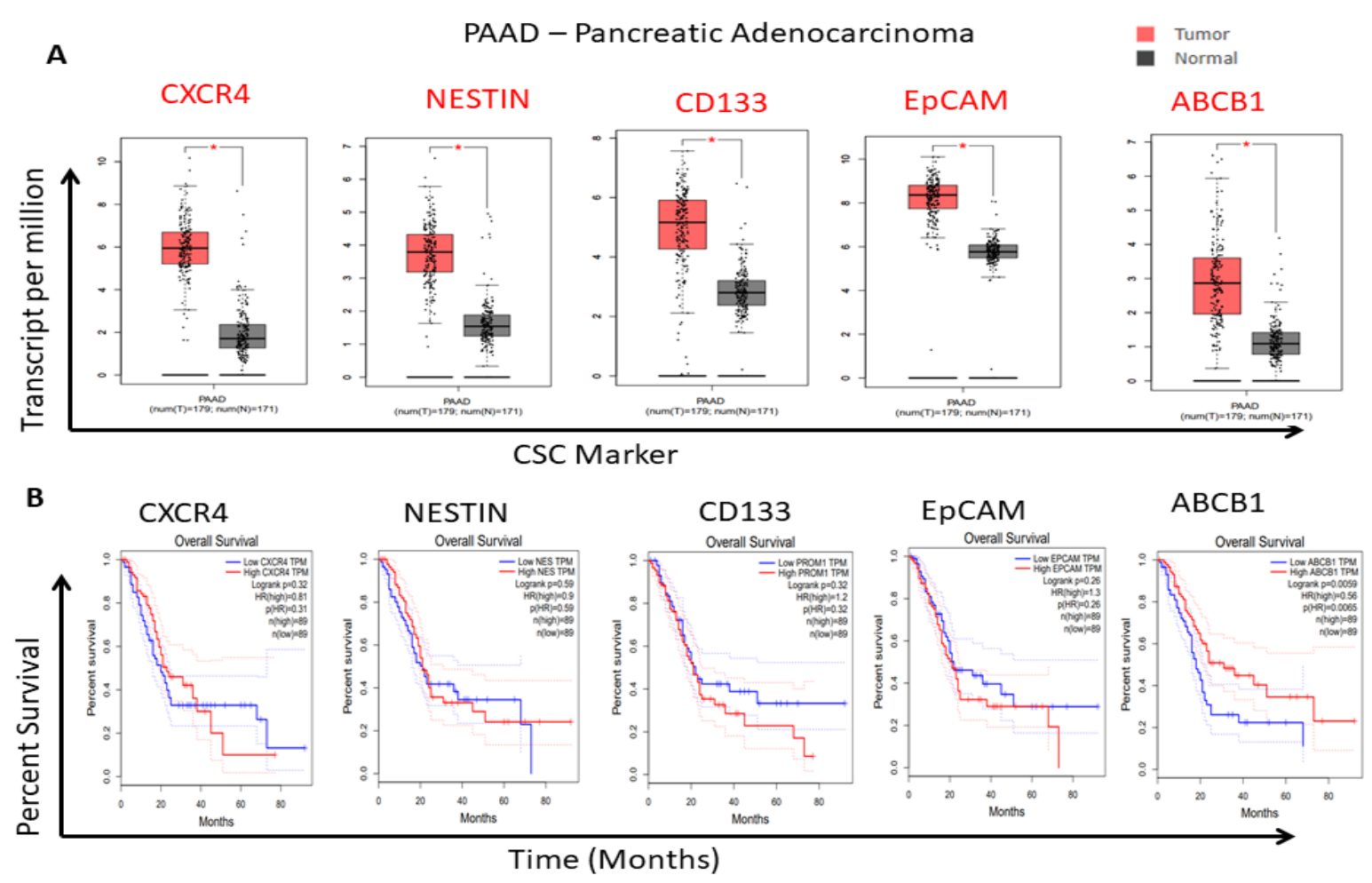

Figure 3. Cancer stem cell markers' gene expression profiles in pancreatic adenocarcinoma (PAAD). The expression of CSC markers CXCR4, Nestin, CD133, EpCAM and ABCB1 in pancreatic adenocarcinoma tissues and adjacent normal tissues (Box plot) based on TCGA/GEPIA database. Data is based on TCGA PAAD samples $n=179$; normal $=171$. CSC markers showing significant differences between tumor and normal samples are shown in red with * indicated for $\mathrm{p}<0.05$ (B) Kaplan-Meier overall survival analysis of PAAD 
patients by CSC markers expression in TCGA data set, high expression $(n=89)$, low expression $(n=89))$.

\subsubsection{Esophageal cancer}

Esophageal cancer is one of the most understudied cancers and has been associated with a poor prognosis $[4,79]$. Several risk factors have been identified but new molecular targets are needed for effective therapy and improvement in patients' outcomes.

Our analysis revealed no difference in expression of commonly used CSC marker, CD44, in tumor tissues compared to normal tissue (Fig 4). Other CSC markers, including ALDH1A1, CD90, ICAM1 and EpCAM showed upregulated expression in ESCA tumor samples versus adjacent normal tissues (Fig 4). Our analysis revealed no association between CSC markers' expression and overall survival between patients with low and high expression of CSC markers (Fig 4).

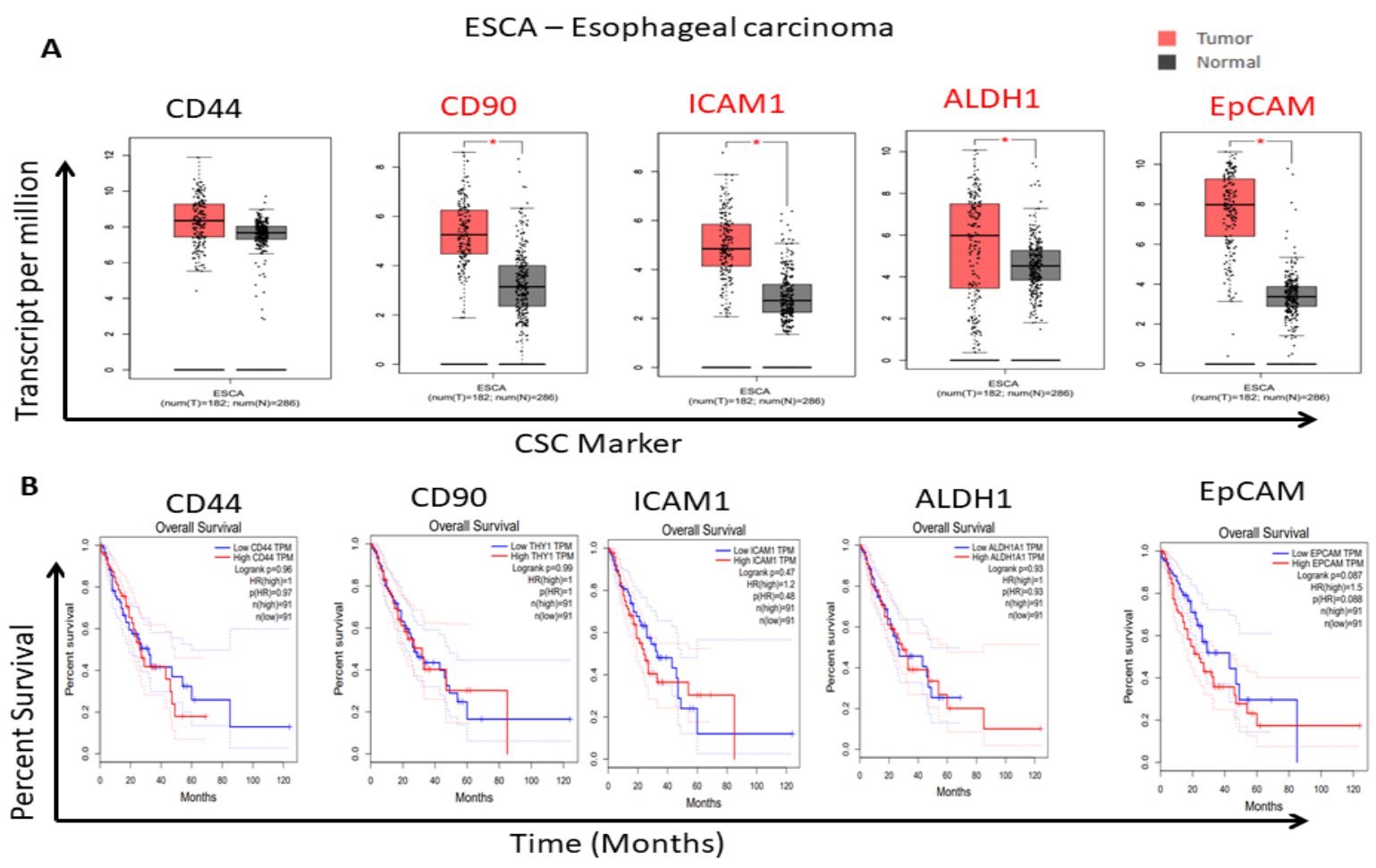


Figure 4. Cancer stem cell markers' gene expression profiles in esophageal carcinoma (ESCA). The expression of CSC markers CD44, CD90, ICAM1, ALDH1 and EpCAM in esophageal carcinoma tissues and adjacent normal tissues (Box plot) based on TCGA/GEPIA database. Data is based on TCGA ESCA samples $n=182$; normal $=286$. CSC markers showing significant differences between tumor and normal samples are shown in red with * indicated for $\mathrm{p}<0.05$ (B) Kaplan-Meier overall survival analysis of ESCA patients by CSC markers expression in TCGA data set (high expression $(n=91)$, low expression $(n=91)$ ).

\subsection{Discussion}

Cancer continues to cause morbidity and mortality globally, with its incidence on the rise in many developing countries. Current cancer therapies eliminate most cells within a tumor but well advanced cancer can progress to drug-resistant disease and metastasis [80]. In addition, tumor heterogeneity contributes to therapy failure and fatal disease outcomes. Several lines of evidence point to therapy itself, especially chemotherapy, causing tumor heterogeneity and thus poor outcomes in patients. Furthermore, according to the CSC theory, most therapies fail to prevent relapse partly due to the presence of a small subpopulation of tumor cells called cancer stem cells $[3,5,6,37,81]$. CSCs reside in the tumor microenvironment and have been proposed to contribute towards the development of therapy resistance and relapse as they can become quiescent. Developing novel strategies against treatment-resistant cancer cells, including CSCs, remains a significant challenge. Despite the considerable progress made in the treatment of cancer in recent years, challenges still remain. Among the many challenges faced in drug development include high cost, low success rates and the poor understanding of the cellular mechanisms driving the disease $[3,81]$. Thus, there is need for new targets and novel drugs or therapies. A new era of CSC-targeted therapies, in combination to conventional therapy, require a deeper understanding of CSCs properties and mechanisms of resistance to therapy. 
Whilst several studies have demonstrated the presence of tumor cells with self-renewal and tumor initiating properties, the identification of markers for such cells is still an ongoing process $[5,17,38,39,82-84]$. Elaborate experiments by Bonnet and Dick revealed that leukamia cancer initiating cells (CSCs) express specific surface markers [85]. In addition, CSCs have been implicated in metastasis. The origin(s) of CSCs is an area under intense scrutiny at the moment with new evidence pointing to normal stem cells [86]. To complicate the matter further, markers for tumor initiating cells are not universal, with CSCs from different tissues expressing different markers. Our bioinformatic analysis showed that CSC markers are mostly highly expressed in patient tumor samples compared to adjacent normal tissues. For example, the expression of CD44, also referred to as Homing Cell Adhesion Molecule (HCAM), was expressed highly in colon adenocarcinoma samples. This suggests that CD44 expression may be used during diagnosis as well as linked to development of therapy resistance. Overall, CD44 may predict COAD prognosis. In many cancers including breast cancer, CD44 together with CD24 are used to isolate and characterise CSCs [5, 46]. The utility of individual CSC markers is not proven hence markers are usually used in combination.

The presence of CSCs in tumors may explain the high occurrence of development of drugresistant disease and relapse $[87,88]$. The results obtained in this analysis clearly suggest novel and better chemotherapeutic drugs must be developed that can provide better efficacy and clinical outcome for cancer patients by targeting not only cancer cells but CSCs as well. In addition, our analysis of the association between CSC marker expression and patients' survival suggest that targeting CSCs using a single marker such as CD44 might not be enough to eradicate cancers. Instead the use of anti-CSC therapy in combination with chemotherapeutic agents could be better at eradicating cancers. For example, monoclonal 
antibodies are one of the new strategies to treat chemo-resistant cancers [89-91]. Due to their specificity, monoclonal antibodies represent a promising method for interfering with a single target molecule with high selectivity [92].

The mechanisms through CSCs develop therapy resistance including chemoresistance have been under intense investigations $[3,5,41,50,53,65,66,77]$. These mechanisms include epithelial mesenchymal transition, quiescence or dormancy, contribution of the tumor microenvironment factors, high expression of drug transporter proteins and enhanced DNA damage repair (Figure 5) [5-7, 53, 77, 93-95]. Markers of EMT and CSCs have been found to be co-expressed, thus linking EMT and CSCs [96]. High expression of drug transporter proteins including $\mathrm{ABCG} 2$ and $\mathrm{ABCB} 1$ allow $\mathrm{CSC}$ to expel chemotherapeutic drugs and thus attain better survival than cancer cells and stromal in a tumor [97].

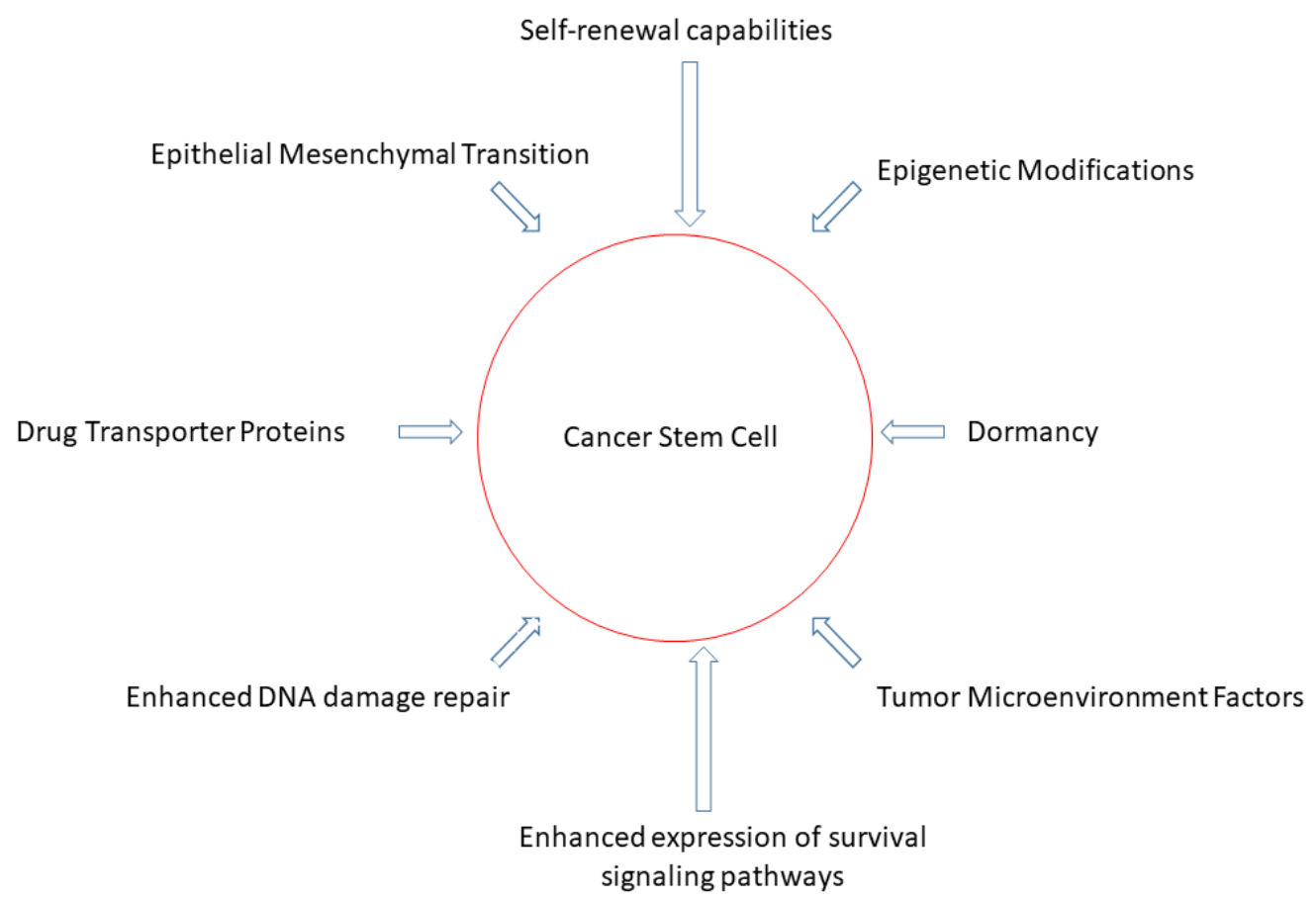

Figure 5. Cancer stem cell properties contributing to development of therapy especially chemoresistance. 
CSCs have been shown to undergo dormancy and during treatment some CSC clones can be induced to grow [98]. In addition CSCs display enhanced reactive oxygen scavenging limiting DNA damage in the process [99]. Tumor microenvironment factors and cells have been shown to aid cancer cells to survive chemotherapy via induction of several survival pathways including the MEK-ERK and TGF- $\beta$ pathway $[8,9,100]$. In addition, hypoxia, cancer-associated fibroblasts and cancer associated macrophages are known to induce stem cell-associated genes, sustaining CSCs within the tumor microenvironment [101-103]. Some of the therapeutic strategies against CSCs include targeting the tumor microenvironment, CSC markers, survival pathways and drug transporters proteins (Figure 6).

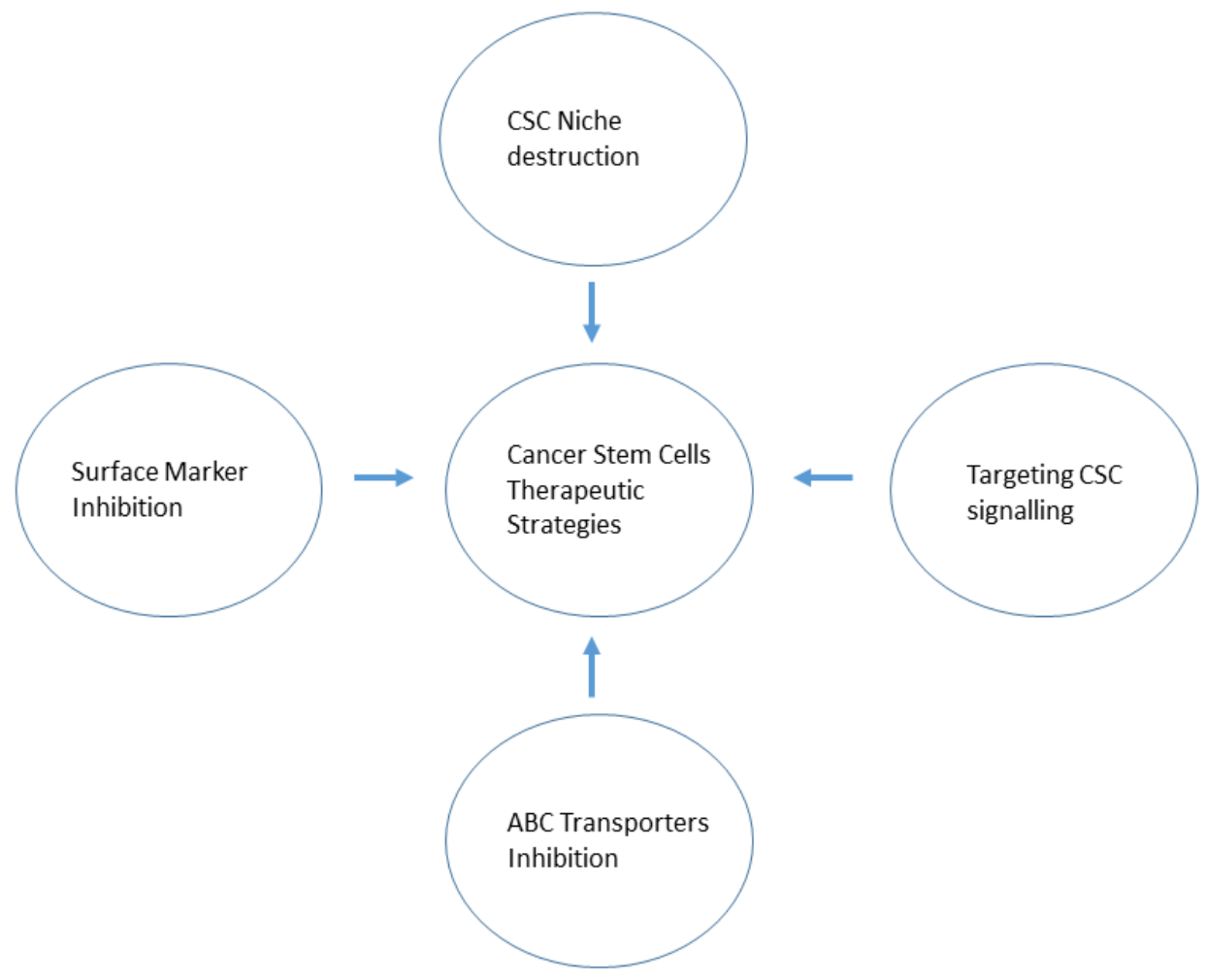

Figure 6. Cancer stem cell-targeted therapies can take the form of surface marker inhibition, drug transporter proteins inhibition, targeting survival signaling pathways and the CSC niche or tumor microenvironment. Figure adapted from Dzobo et al, 2016 [5]. 


\subsection{Study Limitations}

Further study to validate the involvement of these CSC markers in tumor initiation and progression is in progress and much needed. Future studies should carefully validate the results obtained in this study and others to evaluate CSC markers as biomarkers with putative prognostic roles.

\subsection{Conclusions}

This study show that CSC markers are expressed in many cancers and the targeting of these cells would be beneficial to cancer patients. CSC properties are relevant to our understanding of therapy resistance especially chemoresistance. Research into the role of CSCs in cancer initiation and progression holds great potential in the development of novel therapeutic strategies effective in eradicating ESCC. Importantly, this study provides evidence that individual CSC markers may not be useful as valuable predictors of poor prognosis in cancer. Such markers may be useful when used in combinations.

\section{Acknowledgements}

The funding for this research was provided by the National Research Foundation (NRF) of South Africa, South African Medical Research Council, International Centre for Genetic Engineering and Biotechnology (ICGEB) and the University of Cape Town.

\section{Conflict of interest}

The authors declare no conflict of interest 


\section{LIST OF ABBREVIATIONS}

ALDH aldehyde dehydrogenase

ANOVA analysis of variance

CD cluster of differentiation

COAD Colon adenocarcinoma

CSCs Cancer stem cells

ECM Extracellular Matrix

EMT Epithelial to mesenchymal transition

ESCA Esophageal carcinoma

GEPIA Gene expression profiling interactive analysis

HCAM Homing Cell Adhesion Molecule

LUAD Lung adenocarcinoma

MMPs $\quad$ Matrix metalloproteases

MRP2 multidrug resistance protein 2

MSCs Mesenchymal stem cells

PAAD Pancreatic adenocarcinoma

PAAD Pancreatic adenocarcinoma

SCID severe combined immunodefficient 
TCGA The cancer genome atlas

TME Tumor Microenvironment

\section{References}

1. Bray, F.; Ferlay, J.; Soerjomataram, I.; Siegel, R. L.; Torre, L. A.; Jemal, A., Global cancer statistics 2018: GLOBOCAN estimates of incidence and mortality worldwide for 36 cancers in 185 countries. CA: a cancer journal for clinicians 2018, 68, (6), 394-424.

2. The, L., GLOBOCAN 2018: counting the toll of cancer. Lancet (London, England) 2018, 392, (10152), 985.

3. Dzobo, K., Epigenomics-Guided Drug Development: Recent Advances in Solving the Cancer Treatment "jigsaw puzzle". Omics : a journal of integrative biology 2019, 23, (2), 70-85.

4. Dzobo, K.; Hassen, N.; Senthebane, D. A.; Thomford, N. E.; Rowe, A.; Shipanga, H.; Wonkam, A.; Parker, M. I.; Mowla, S.; Dandara, C., Chemoresistance to Cancer Treatment: Benzoalpha-Pyrene as Friend or Foe? Molecules (Basel, Switzerland) 2018, 23, (4).

5. Dzobo, K.; Senthebane, D. A.; Rowe, A.; Thomford, N. E.; Mwapagha, L. M.; Al-Awwad, N.; Dandara, C.; Parker, M. I., Cancer Stem Cell Hypothesis for Therapeutic Innovation in Clinical Oncology? Taking the Root Out, Not Chopping the Leaf. Omics : a journal of integrative biology 2016, 20, (12), 681-691.

6. Dzobo, K.; Senthebane, D. A.; Thomford, N. E.; Rowe, A.; Dandara, C.; Parker, M. I., Not Everyone Fits the Mold: Intratumor and Intertumor Heterogeneity and Innovative Cancer Drug Design and Development. Omics : a journal of integrative biology 2018, 22, (1), 17-34.

7. Easwaran, H.; Tsai, H. C.; Baylin, S. B., Cancer epigenetics: tumor heterogeneity, plasticity of stem-like states, and drug resistance. Molecular cell 2014, 54, (5), 716-27.

8. Senthebane, D.; Jonker, T.; Rowe, A.; Thomford, N.; Munro, D.; Dandara, C.; Wonkam, A.; Govender, D.; Calder, B.; Soares, N., The role of tumor microenvironment in chemoresistance: 3D extracellular matrices as accomplices. International journal of molecular sciences 2018, 19, (10), 2861.

9. Senthebane, D. A.; Rowe, A.; Thomford, N. E.; Shipanga, H.; Munro, D.; Al Mazeedi, M. A.; Almazyadi, H. A.; Kallmeyer, K.; Dandara, C.; Pepper, M. S., The role of tumor microenvironment in chemoresistance: to survive, keep your enemies closer. International journal of molecular sciences 2017, 18, (7), 1586.

10. Bai, X.; Chen, Y.; Hou, X.; Huang, M.; Jin, J., Emerging role of NRF2 in chemoresistance by regulating drug-metabolizing enzymes and efflux transporters. Drug Metab Rev 2016, 48, (4), 541-567.

11. Begicevic, R. R.; Falasca, M., ABC Transporters in Cancer Stem Cells: Beyond Chemoresistance. International journal of molecular sciences 2017, 18, (11).

12. Bhatt, V. R.; Vose, J. M., Hematopoietic stem cell transplantation for non-Hodgkin lymphoma. Hematology/oncology clinics of North America 2014, 28, (6), 1073-95.

13. Borley, J.; Brown, R., Epigenetic mechanisms and therapeutic targets of chemotherapy resistance in epithelial ovarian cancer. Ann Med 2015, 47, (5), 359-69.

14. Brunen, D.; Willems, S. M.; Kellner, U.; Midgley, R.; Simon, I.; Bernards, R., TGF-beta: an emerging player in drug resistance. Cell cycle (Georgetown, Tex.) 2013, 12, (18), 2960-8.

15. Dzobo, K., Taking a Full Snapshot of Cancer Biology: Deciphering the Tumor Microenvironment for Effective Cancer Therapy in the Oncology Clinic. Omics : a journal of integrative biology 2020. 
16. Sheehan, K. M.; Sheahan, K.; O'Donoghue, D. P.; MacSweeney, F.; Conroy, R. M.; Fitzgerald, D. J.; Murray, F. E., The relationship between cyclooxygenase-2 expression and colorectal cancer. Jama 1999, 282, (13), 1254-7.

17. Curtarelli, R. B.; Goncalves, J. M.; Dos Santos, L. G. P.; Savi, M. G.; Nor, J. E.; Mezzomo, L. A. M.; Rodriguez Cordeiro, M. M., Expression of Cancer Stem Cell Biomarkers in Human Head and Neck Carcinomas: a Systematic Review. Stem cell reviews 2018, 14, (6), 769-784.

18. Lee, J. S.; Kim, W. G., Potential protective effect of ALDH-1 stromal expression against early recurrence of operable breast cancers. Stem Cell Investig 2018, 5, 43.

19. Mohamed, H. T.; Gadalla, R.; El-Husseiny, N.; Hassan, H.; Wang, Z.; Ibrahim, S. A.; El-Shinawi, M.; Sherr, D. H.; Mohamed, M. M., Inflammatory breast cancer: Activation of the aryl hydrocarbon receptor and its target CYP1B1 correlates closely with Wnt5a/b-beta-catenin signalling, the stem cell phenotype and disease progression. J Adv Res 2019, 16, 75-86.

20. Riaz, N.; Idress, R.; Habib, S.; Azam, I.; Lalani, E. M., Expression of Androgen Receptor and Cancer Stem Cell Markers (CD44(+)/CD24(-) and ALDH1(+)): Prognostic Implications in Invasive Breast Cancer. Trans/ Oncol 2018, 11, (4), 920-929.

21. Tao, Y.; Li, H.; Huang, R.; Mo, D.; Zeng, T.; Fang, M.; Li, M., Clinicopathological and Prognostic Significance of Cancer Stem Cell Markers in Ovarian Cancer Patients: Evidence from 52 Studies. Cellular physiology and biochemistry : international journal of experimental cellular physiology, biochemistry, and pharmacology 2018, 46, (4), 1716-1726.

22. Yang, Z.; Ni, W.; Cui, C.; Qi, W.; Piao, L.; Xuan, Y., Identification of LETM1 as a marker of cancer stem-like cells and predictor of poor prognosis in esophageal squamous cell carcinoma. Human pathology 2018, 81, 148-156.

23. Collina, F.; Di Bonito, M.; Li Bergolis, V.; De Laurentiis, M.; Vitagliano, C.; Cerrone, M.; Nuzzo, F.; Cantile, M.; Botti, G., Prognostic Value of Cancer Stem Cells Markers in Triple-Negative Breast Cancer. BioMed research international 2015, 2015, 158682.

24. Saeg, F.; Anbalagan, M., Breast cancer stem cells and the challenges of eradication: a review of novel therapies. Stem Cell Investig 2018, 5, 39.

25. Dong, C.; Cui, D.; Liu, G.; Xu, H.; Peng, X.; Duan, J.; Liu, L., Cancer stem cell associated eight gene-based signature predicts clinical outcomes of colorectal cancer. Oncology letters 2019, $17,(1), 442-449$.

26. Mohamed, H.; Hagstrom, J.; Jouhi, L.; Atula, T.; Almangush, A.; Makitie, A.; Haglund, C., The expression and prognostic value of stem cell markers Bmi-1, HESC5:3, and HES77 in human papillomavirus-positive and -negative oropharyngeal squamous cell carcinoma. Tumour biology : the journal of the International Society for Oncodevelopmental Biology and Medicine 2019, 41, (3), 1010428319840473.

27. Pece, S.; Disalvatore, D.; Tosoni, D.; Vecchi, M.; Confalonieri, S.; Bertalot, G.; Viale, G.; Colleoni, M.; Veronesi, P.; Galimberti, V.; Di Fiore, P. P., Identification and clinical validation of a multigene assay that interrogates the biology of cancer stem cells and predicts metastasis in breast cancer: A retrospective consecutive study. EBioMedicine 2019.

28. Wang, J. H.; Huang, S. T.; Zhang, L.; Liu, Z. G.; Liang, R. X.; Jiang, S. W.; Jiang, Y. N.; Yu, X. J.; Jiang, Y. C.; Li, X. Z.; Zhang, P. F.; Wen, Z. S.; Zheng, M., Combined prognostic value of the cancer stem cell markers CD47 and CD133 in esophageal squamous cell carcinoma. Cancer Med 2019, 8, (3), 1315-1325.

29. Devesa, S. S.; Blot, W. J.; Fraumeni, J. F., Jr., Changing patterns in the incidence of esophageal and gastric carcinoma in the United States. Cancer 1998, 83, (10), 2049-53.

30. Maugeri-Sacca, M.; Vigneri, P.; De Maria, R., Cancer stem cells and chemosensitivity. Clinical cancer research : an official journal of the American Association for Cancer Research 2011, 17, (15), 4942-7.

31. Rice, T. W.; Adelstein, D. J.; Zuccaro, G.; Falk, G. W.; Goldblum, J. R., Advances in the treatment of esophageal carcinoma. The Gastroenterologist 1997, 5, (4), 278-94. 
32. Zhang, C.; Ma, Q.; Shi, Y.; Li, X.; Wang, M.; Wang, J.; Ge, J.; Chen, Z.; Wang, Z.; Jiang, H., A novel 5 -fluorouracil-resistant human esophageal squamous cell carcinoma cell line Eca109/5-FU with significant drug resistance-related characteristics. Oncology reports 2017, 37, (5), 2942-2954.

33. Hamano, R.; Miyata, H.; Yamasaki, M.; Kurokawa, Y.; Hara, J.; Moon, J. H.; Nakajima, K.; Takiguchi, S.; Fujiwara, Y.; Mori, M.; Doki, Y., Overexpression of miR-200c induces chemoresistance in esophageal cancers mediated through activation of the Akt signaling pathway. Clinical cancer research : an official journal of the American Association for Cancer Research 2011, 17, (9), 3029-38.

34. Vermorken, J. B.; Herbst, R. S.; Leon, X.; Amellal, N.; Baselga, J., Overview of the efficacy of cetuximab in recurrent and/or metastatic squamous cell carcinoma of the head and neck in patients who previously failed platinum-based therapies. Cancer 2008, 112, (12), 2710-9.

35. Vulsteke, C.; Lambrechts, D.; Dieudonne, A.; Hatse, S.; Brouwers, B.; van Brussel, T.; Neven, P.; Belmans, A.; Schoffski, P.; Paridaens, R.; Wildiers, H., Genetic variability in the multidrug resistance associated protein-1 ( $A B C C 1 / M R P 1)$ predicts hematological toxicity in breast cancer patients receiving (neo-)adjuvant chemotherapy with 5-fluorouracil, epirubicin and cyclophosphamide (FEC). Annals of oncology : official journal of the European Society for Medical Oncology 2013, 24, (6), 1513-25.

36. Yang, H.; Li, X. D.; Zhou, Y.; Ban, X.; Zeng, T. T.; Li, L.; Zhang, B. Z.; Yun, J.; Xie, D.; Guan, X. Y.; $\mathrm{Li}, \mathrm{Y}$., Stemness and chemotherapeutic drug resistance induced by EIF5A2 overexpression in esophageal squamous cell carcinoma. Oncotarget 2015, 6, (28), 26079-89.

37. Lapidot, T.; Sirard, C.; Vormoor, J.; Murdoch, B.; Hoang, T.; Caceres-Cortes, J.; Minden, M.; Paterson, B.; Caligiuri, M. A.; Dick, J. E., A cell initiating human acute myeloid leukaemia after transplantation into SCID mice. Nature 1994, 367, (6464), 645-8.

38. Ahmad, G.; Amiji, M. M., Cancer stem cell-targeted therapeutics and delivery strategies. Expert Opin Drug Deliv 2017, 14, (8), 997-1008.

39. Almanaa, T. N.; Geusz, M. E.; Jamasbi, R. J., A new method for identifying stem-like cells in esophageal cancer cell lines. Journal of Cancer 2013, 4, (7), 536-48.

40. Cabrera, M. C.; Hollingsworth, R. E.; Hurt, E. M., Cancer stem cell plasticity and tumor hierarchy. World J Stem Cells 2015, 7, (1), 27-36.

41. Geng, S. Q.; Alexandrou, A. T.; Li, J. J., Breast cancer stem cells: Multiple capacities in tumor metastasis. Cancer letters 2014, 349, (1), 1-7.

42. Kise, K.; Kinugasa-Katayama, Y.; Takakura, N., Tumor microenvironment for cancer stem cells. Adv Drug Deliv Rev 2016, 99, (Pt B), 197-205.

43. MacDonagh, L.; Gray, S. G.; Breen, E.; Cuffe, S.; Finn, S. P.; O'Byrne, K. J.; Barr, M. P., Lung cancer stem cells: The root of resistance. Cancer letters 2016, 372, (2), 147-56.

44. Savage, P., Chemotherapy curable malignancies and cancer stem cells: a biological review and hypothesis. BMC cancer 2016, 16, (1), 906.

45. Prince, M. E.; Sivanandan, R.; Kaczorowski, A.; Wolf, G. T.; Kaplan, M. J.; Dalerba, P.; Weissman, I. L.; Clarke, M. F.; Ailles, L. E., Identification of a subpopulation of cells with cancer stem cell properties in head and neck squamous cell carcinoma. Proceedings of the National Academy of Sciences of the United States of America 2007, 104, (3), 973-8.

46. Al-Hajj, M.; Wicha, M. S.; Benito-Hernandez, A.; Morrison, S. J.; Clarke, M. F., Prospective identification of tumorigenic breast cancer cells. Proceedings of the National Academy of Sciences of the United States of America 2003, 100, (7), 3983-8.

47. Bodenstine, T. M.; Chandler, G. S.; Seftor, R. E.; Seftor, E. A.; Hendrix, M. J., Plasticity underlies tumor progression: role of Nodal signaling. Cancer metastasis reviews 2016, 35, (1), 21-39.

48. Caiado, F.; Silva-Santos, B.; Norell, H., Intra-tumour heterogeneity - going beyond genetics. The FEBS journal 2016, 283, (12), 2245-58. 
49. Cortina, C.; Turon, G.; Stork, D.; Hernando-Momblona, X.; Sevillano, M.; Aguilera, M.; Tosi, S.; Merlos-Suarez, A.; Stephan-Otto Attolini, C.; Sancho, E.; Batlle, E., A genome editing approach to study cancer stem cells in human tumors. EMBO molecular medicine 2017, 9, (7), 869-879.

50. Ishizawa, K.; Rasheed, Z. A.; Karisch, R.; Wang, Q.; Kowalski, J.; Susky, E.; Pereira, K.; Karamboulas, C.; Moghal, N.; Rajeshkumar, N. V.; Hidalgo, M.; Tsao, M.; Ailles, L.; Waddell, T. K.; Maitra, A.; Neel, B. G.; Matsui, W., Tumor-initiating cells are rare in many human tumors. Cell stem cell 2010, 7, (3), 279-82.

51. Schatton, T.; Murphy, G. F.; Frank, N. Y.; Yamaura, K.; Waaga-Gasser, A. M.; Gasser, M.; Zhan, Q.; Jordan, S.; Duncan, L. M.; Weishaupt, C.; Fuhlbrigge, R. C.; Kupper, T. S.; Sayegh, M. H.; Frank, M. H., Identification of cells initiating human melanomas. Nature 2008, 451, (7176), 345-9.

52. Zabierowski, S. E.; Herlyn, M., Learning the ABCs of melanoma-initiating cells. Cancer cell 2008, 13, (3), 185-7.

53. Fan, C. W.; Chen, T.; Shang, Y. N.; Gu, Y. Z.; Zhang, S. L.; Lu, R.; OuYang, S. R.; Zhou, X.; Li, Y.; Meng, W. T.; Hu, J. K.; Lu, Y.; Sun, X. F.; Bu, H.; Zhou, Z. G.; Mo, X. M., Cancer-initiating cells derived from human rectal adenocarcinoma tissues carry mesenchymal phenotypes and resist drug therapies. Cell death \& disease 2013, 4, e828.

54. Ricci-Vitiani, L.; Lombardi, D. G.; Pilozzi, E.; Biffoni, M.; Todaro, M.; Peschle, C.; De Maria, R., Identification and expansion of human colon-cancer-initiating cells. Nature 2007, 445, (7123), 111-5.

55. Britton, K. M.; Kirby, J. A.; Lennard, T. W.; Meeson, A. P., Cancer stem cells and side population cells in breast cancer and metastasis. Cancers 2011, 3, (2), 2106-30.

56. Boesch, M.; Zeimet, A. G.; Reimer, D.; Schmidt, S.; Gastl, G.; Parson, W.; Spoeck, F.; Hatina, J.; Wolf, D.; Sopper, S., The side population of ovarian cancer cells defines a heterogeneous compartment exhibiting stem cell characteristics. Oncotarget 2014, 5, (16), 7027-39.

57. Szotek, P. P.; Pieretti-Vanmarcke, R.; Masiakos, P. T.; Dinulescu, D. M.; Connolly, D.; Foster, R.; Dombkowski, D.; Preffer, F.; Maclaughlin, D. T.; Donahoe, P. K., Ovarian cancer side population defines cells with stem cell-like characteristics and Mullerian Inhibiting Substance responsiveness. Proceedings of the National Academy of Sciences of the United States of America 2006, 103, (30), 11154-9.

58. Kobayashi, T.; Masutomi, K.; Tamura, K.; Moriya, T.; Yamasaki, T.; Fujiwara, Y.; Takahashi, S.; Yamamoto, J.; Tsuda, H., Nucleostemin expression in invasive breast cancer. BMC cancer 2014, 14, 215.

59. Takahashi, R. U.; Takeshita, F.; Fujiwara, T.; Ono, M.; Ochiya, T., Cancer stem cells in breast cancer. Cancers 2011, 3, (1), 1311-28.

60. Fujiwara, T.; Katsuda, T.; Hagiwara, K.; Kosaka, N.; Yoshioka, Y.; Takahashi, R. U.; Takeshita, F.; Kubota, D.; Kondo, T.; Ichikawa, H.; Yoshida, A.; Kobayashi, E.; Kawai, A.; Ozaki, T.; Ochiya, T., Clinical relevance and therapeutic significance of microRNA-133a expression profiles and functions in malignant osteosarcoma-initiating cells. Stem cells (Dayton, Ohio) 2014, 32, (4), 959-73.

61. Fujiwara, T.; Ozaki, T., Overcoming Therapeutic Resistance of Bone Sarcomas: Overview of the Molecular Mechanisms and Therapeutic Targets for Bone Sarcoma Stem Cells. Stem cells international 2016, 2016, 2603092.

62. Zhang, G.; Ma, L.; Xie, Y. K.; Miao, X. B.; Jin, C., Esophageal cancer tumorspheres involve cancer stem-like populations with elevated aldehyde dehydrogenase enzymatic activity. Molecular medicine reports 2012, 6, (3), 519-24.

63. Gangavarapu, K. J.; Azabdaftari, G.; Morrison, C. D.; Miller, A.; Foster, B. A.; Huss, W. J., Aldehyde dehydrogenase and ATP binding cassette transporter G2 (ABCG2) functional assays isolate different populations of prostate stem cells where ABCG2 function selects for cells with increased stem cell activity. Stem cell research \& therapy 2013, 4, (5), 132. 
64. Richard, V.; Nair, M. G.; Santhosh Kumar, T. R.; Pillai, M. R., Side population cells as prototype of chemoresistant, tumor-initiating cells. BioMed research international 2013, 2013, 517237.

65. Haraguchi, N.; Inoue, H.; Tanaka, F.; Mimori, K.; Utsunomiya, T.; Sasaki, A.; Mori, M., Cancer stem cells in human gastrointestinal cancers. Human cell 2006, 19, (1), 24-9.

66. Haraguchi, N.; Utsunomiya, T.; Inoue, H.; Tanaka, F.; Mimori, K.; Barnard, G. F.; Mori, M., Characterization of a side population of cancer cells from human gastrointestinal system. Stem cells (Dayton, Ohio) 2006, 24, (3), 506-13.

67. Ferlay, J.; Colombet, M.; Soerjomataram, I.; Mathers, C.; Parkin, D. M.; Pineros, M.; Znaor, A.; Bray, F., Estimating the global cancer incidence and mortality in 2018: GLOBOCAN sources and methods. International journal of cancer 2019, 144, (8), 1941-1953.

68. Gerli, M. F. M.; Guyette, J. P.; Evangelista-Leite, D.; Ghoshhajra, B. B.; Ott, H. C., Perfusion decellularization of a human limb: A novel platform for composite tissue engineering and reconstructive surgery. PLoS One 2018, 13, (1), e0191497.

69. Torre, L. A.; Bray, F.; Siegel, R. L.; Ferlay, J.; Lortet-Tieulent, J.; Jemal, A., Global cancer statistics, 2012. CA: a cancer journal for clinicians 2015, 65, (2), 87-108.

70. Merritt, E. K.; Hammers, D. W.; Tierney, M.; Suggs, L. J.; Walters, T. J.; Farrar, R. P., Functional assessment of skeletal muscle regeneration utilizing homologous extracellular matrix as scaffolding. Tissue engineering. Part A 2010, 16, (4), 1395-405.

71. Sicari, B. M.; Rubin, J. P.; Dearth, C. L.; Wolf, M. T.; Ambrosio, F.; Boninger, M.; Turner, N. J.; Weber, D. J.; Simpson, T. W.; Wyse, A.; Brown, E. H.; Dziki, J. L.; Fisher, L. E.; Brown, S.; Badylak, S. F., An acellular biologic scaffold promotes skeletal muscle formation in mice and humans with volumetric muscle loss. Science translational medicine 2014, 6, (234), 234 ra58.

72. Ansari, D.; Tingstedt, B.; Andersson, B.; Holmquist, F.; Sturesson, C.; Williamsson, C.; Sasor, A.; Borg, D.; Bauden, M.; Andersson, R., Pancreatic cancer: yesterday, today and tomorrow. Future oncology (London, England) 2016, 12, (16), 1929-46.

73. McGuigan, A.; Kelly, P.; Turkington, R. C.; Jones, C.; Coleman, H. G.; McCain, R. S., Pancreatic cancer: A review of clinical diagnosis, epidemiology, treatment and outcomes. World journal of gastroenterology 2018, 24, (43), 4846-4861.

74. Tian, C.; Clauser, K. R.; Öhlund, D.; Rickelt, S.; Huang, Y.; Gupta, M.; Mani, D. R.; Carr, S. A.; Tuveson, D. A.; Hynes, R. O., Proteomic analyses of ECM during pancreatic ductal adenocarcinoma progression reveal different contributions by tumor and stromal cells. Proceedings of the National Academy of Sciences of the United States of America 2019, 116, (39), 19609-19618.

75. Zheng, L.; Xue, J.; Jaffee, E. M.; Habtezion, A., Role of immune cells and immune-based therapies in pancreatitis and pancreatic ductal adenocarcinoma. Gastroenterology 2013, 144, (6), 1230-40.

76. Chu, L. C.; Goggins, M. G.; Fishman, E. K., Diagnosis and Detection of Pancreatic Cancer. Cancer journal (Sudbury, Mass.) 2017, 23, (6), 333-342.

77. Ercan, G.; Karlitepe, A.; Ozpolat, B., Pancreatic Cancer Stem Cells and Therapeutic Approaches. Anticancer research 2017, 37, (6), 2761-2775.

78. Ilic, M.; Ilic, I., Epidemiology of pancreatic cancer. World journal of gastroenterology 2016, 22, (44), 9694-9705.

79. Dzobo, K.; Vogelsang, M.; Thomford, N. E.; Dandara, C.; Kallmeyer, K.; Pepper, M. S.; Parker, M. I., Wharton's Jelly-Derived Mesenchymal Stromal Cells and Fibroblast-Derived Extracellular Matrix Synergistically Activate Apoptosis in a p21-Dependent Mechanism in WHCO1 and MDA MB 231 Cancer Cells In Vitro. Stem cells international 2016, 2016, 4842134.

80. Chan, D.; Zhou, Y.; Chui, C. H.; Lam, K. H.; Law, S.; Chan, A. S.; Li, X.; Lam, A. K.; Tang, J. C. O., Expression of Insulin-Like Growth Factor Binding Protein-5 (IGFBP5) Reverses CisplatinResistance in Esophageal Carcinoma. Cells 2018, 7, (10). 
81. Dzobo, K.; Rowe, A.; Senthebane, D. A.; AlMazyadi, M. A. M.; Patten, V.; Parker, M. I., ThreeDimensional Organoids in Cancer Research: The Search for the Holy Grail of Preclinical Cancer Modeling. Omics : a journal of integrative biology 2018, 22, (12), 733-748.

82. Albini, A.; Bruno, A.; Gallo, C.; Pajardi, G.; Noonan, D. M.; Dallaglio, K., Cancer stem cells and the tumor microenvironment: interplay in tumor heterogeneity. Connective tissue research 2015, 56, (5), 414-25.

83. Assenov, Y.; Brocks, D.; Gerhauser, C., Intratumor heterogeneity in epigenetic patterns. Seminars in cancer biology 2018.

84. La Porta, C. A. M.; Zapperi, S., Complexity in cancer stem cells and tumor evolution: Toward precision medicine. Seminars in cancer biology 2017, 44, 3-9.

85. Bonnet, D.; Dick, J. E., Human acute myeloid leukemia is organized as a hierarchy that originates from a primitive hematopoietic cell. Nature medicine 1997, 3, (7), 730-7.

86. Afify, S. M.; Seno, M., Conversion of stem cells to cancer stem cells: undercurrent of cancer initiation. Cancers 2019, 11, (3), 345.

87. Wang, D.; Plukker, J. T. M.; Coppes, R. P., Cancer stem cells with increased metastatic potential as a therapeutic target for esophageal cancer. Seminars in cancer biology 2017, 44, 60-66.

88. Wang, X.; Cao, Y.; Zhang, S.; Chen, Z.; Fan, L.; Shen, X.; Zhou, S.; Chen, D., Stem cell autocrine CXCL12/CXCR4 stimulates invasion and metastasis of esophageal cancer. Oncotarget 2017, 8, (22), 36149-36160.

89. Cremolini, C.; Morano, F.; Moretto, R.; Berenato, R.; Tamborini, E.; Perrone, F.; Rossini, D.; Gloghini, A.; Busico, A.; Zucchelli, G.; Baratelli, C.; Tamburini, E.; Tampellini, M.; Sensi, E.; Fuca, G.; Volpi, C.; Milione, M.; Di Maio, M.; Fontanini, G.; De Braud, F.; Falcone, A.; Pietrantonio, F., Negative hyper-selection of metastatic colorectal cancer patients for antiEGFR monoclonal antibodies: the PRESSING case-control study. Annals of oncology : official journal of the European Society for Medical Oncology 2017, 28, (12), 3009-3014.

90. Lambert, J. M.; Morris, C. Q., Antibody-Drug Conjugates (ADCs) for Personalized Treatment of Solid Tumors: A Review. Advances in therapy 2017, 34, (5), 1015-1035.

91. Topalian, S. L.; Hodi, F. S.; Brahmer, J. R.; Gettinger, S. N.; Smith, D. C.; McDermott, D. F.; Powderly, J. D.; Carvajal, R. D.; Sosman, J. A.; Atkins, M. B.; Leming, P. D.; Spigel, D. R.; Antonia, S. J.; Horn, L.; Drake, C. G.; Pardoll, D. M.; Chen, L.; Sharfman, W. H.; Anders, R. A.; Taube, J. M.; McMiller, T. L.; Xu, H.; Korman, A. J.; Jure-Kunkel, M.; Agrawal, S.; McDonald, D.; Kollia, G. D.; Gupta, A.; Wigginton, J. M.; Sznol, M., Safety, activity, and immune correlates of anti-PD-1 antibody in cancer. The New England journal of medicine 2012, 366, (26), 2443-54.

92. Purow, B.; Staveley-O'Carroll, K., Targeting of vaccinia virus using biotin-avidin viral coating and biotinylated antibodies. The Journal of surgical research 2005, 123, (1), 49-54.

93. Dandara, C.; Robertson, B.; Dzobo, K.; Moodley, L.; Parker, M. I., Patient and tumour characteristics as prognostic markers for oesophageal cancer: a retrospective analysis of a cohort of patients at Groote Schuur Hospital. European journal of cardio-thoracic surgery : official journal of the European Association for Cardio-thoracic Surgery 2016, 49, (2), 629-34.

94. Dzobo, K.; Hassen, N.; Senthebane, D. A.; Thomford, N. E.; Rowe, A.; Shipanga, H.; Wonkam, A.; Parker, M. I.; Mowla, S.; Dandara, C., Chemoresistance to Cancer Treatment: Benzo- $\alpha$ Pyrene as Friend or Foe? Molecules (Basel, Switzerland) 2018, 23, (4).

95. Vogelsang, M.; Paccez, J. D.; Schäfer, G.; Dzobo, K.; Zerbini, L. F.; Parker, M. I., Aberrant methylation of the MSH3 promoter and distal enhancer in esophageal cancer patients exposed to first-hand tobacco smoke. Journal of cancer research and clinical oncology 2014, 140, (11), 1825-33.

96. Oskarsson, T.; Batlle, E.; Massagué, J., Metastatic stem cells: sources, niches, and vital pathways. Cell stem cell 2014, 14, (3), 306-321. 
97. Hirschmann-Jax, C.; Foster, A. E.; Wulf, G. G.; Nuchtern, J. G.; Jax, T. W.; Gobel, U.; Goodell, M. A.; Brenner, M. K., A distinct "side population" of cells with high drug efflux capacity in human tumor cells. Proceedings of the National Academy of Sciences 2004, 101, (39), 1422814233.

98. Kreso, A.; O'Brien, C. A.; Van Galen, P.; Gan, O. I.; Notta, F.; Brown, A. M.; Ng, K.; Ma, J.; Wienholds, E.; Dunant, C., Variable clonal repopulation dynamics influence chemotherapy response in colorectal cancer. Science (New York, N.Y.) 2013, 339, (6119), 543-548.

99. Peitzsch, C.; Kurth, I.; Kunz-Schughart, L.; Baumann, M.; Dubrovska, A., Discovery of the cancer stem cell related determinants of radioresistance. Radiotherapy and Oncology 2013, $108,(3), 378-387$.

100. Lu, J.; Ye, X.; Fan, F.; Xia, L.; Bhattacharya, R.; Bellister, S.; Tozzi, F.; Sceusi, E.; Zhou, Y.; Tachibana, I., Endothelial cells promote the colorectal cancer stem cell phenotype through a soluble form of Jagged-1. Cancer cell 2013, 23, (2), 171-185.

101. Bao, B.; Ali, S.; Ahmad, A.; Azmi, A. S.; Li, Y.; Banerjee, S.; Kong, D.; Sethi, S.; Aboukameel, A.; Padhye, S. B., Hypoxia-induced aggressiveness of pancreatic cancer cells is due to increased expression of VEGF, IL- 6 and miR-21, which can be attenuated by CDF treatment. PloS one 2012, 7, (12).

102. Lotti, F.; Jarrar, A. M.; Pai, R. K.; Hitomi, M.; Lathia, J.; Mace, A.; Gantt Jr, G. A.; Sukhdeo, K.; DeVecchio, J.; Vasanji, A., Chemotherapy activates cancer-associated fibroblasts to maintain colorectal cancer-initiating cells by IL-17A. Journal of Experimental Medicine 2013, 210, (13), 2851-2872.

103. Zheng, Y.; Cai, Z.; Wang, S.; Zhang, X.; Qian, J.; Hong, S.; Li, H.; Wang, M.; Yang, J.; Yi, Q., Macrophages are an abundant component of myeloma microenvironment and protect myeloma cells from chemotherapy drug-induced apoptosis. Blood, The Journal of the American Society of Hematology 2009, 114, (17), 3625-3628. 